\title{
Editorial
}

\section{Renal disease with OCRL1 mutations: Dent-2 or Lowe syndrome?}

\author{
Nomy Levin-Iaina and Dganit Dinour* \\ Nephrology and Hypertension Institute, The Chaim Sheba Medical Center, Tel-Aviv University, Sackler School of \\ Medicine, Tel-Hashomer, Israel
}

Received 9 February 2011

Accepted 9 February 2011

Dent disease is an X-linked tubulopathy, characterized by low molecular weight proteinuria, hypercalciuria, nephrocalcinosis and nephrolithiasis that may progress to advanced renal failure [1,2]. During the last decade, loss-of-function mutations of the CLCN5 gene, which is located in chromosome Xp11.22 and encodes the renal chloride/proton antiporter $\mathrm{ClC}-5$, have been consistently reported in patients with Dent disease [3]. However, in about $40 \%$ of patients with a Dent-like phenotype, no CLCN5 mutations are found [4]. In 2005, Hoopes et al. [4] showed for the first time that the Dent phenotype may also be caused by mutations in the OCRL1 gene, which encodes a phophatidylinositol 4,5-phosphate (PIP2) 5-phosphatase, located in the trans-Golgi network. These findings were confirmed by other reports [4-6] and led to the definition of two types of Dent disease: Dent-1, caused by CLCN5 mutations (OMIM \#300009) and Dent-2, caused by OCRL1 mutations (OMIM \#300555), which accounts for about 15-20\% of Dent cases [7].

PIP2 5-phosphatase, which is encoded by the OCRL1 gene, is expressed ubiquitously in human tissues, including the eyes, kidneys and brain, the main organs involved in Lowe syndrome. This protein is distributed predominantly in the Golgi complex, lysosomes and endosomes [8]. Both $\mathrm{ClC}-5$ and PIP2

*Corresponding author: Dganit Dinour, Department of Nephrology and Hypertension, The Chaim Sheba Medical Center, Tel-Hashomer, Israel. E-mail: Dganit.Dinour@sheba.health.gov.il. 5-phosphatase are expressed in endosomes of the proximal tubular cells and thought to be related to the recycling of multi-ligand receptors, such as megalin and cubilin, which are commonly involved in both Dent-1 and Dent-2 disease. Further genetic heterogeneity is assumed to exist, since there are patients expressing the distinctive phenotype of Dent disease, in which no mutation was identified in either CLCN5 or OCRL1 genes [4].

The OCRL1 gene is also mutated in the oculocerebrorenal syndrome of Lowe (OMIM \#309000), which is a rare $\mathrm{X}$-linked disorder, characterized by bilateral congenital cataracts, mental retardation and a selective proximal tubular dysfunction very similar to the renal phenotype of patients with Dent disease [9]. However, progressive renal failure is common, and is typically more aggressive and occurs at an earlier age than in Dent- 2 disease. Thus, loss of Ocrl1 function can cause a spectrum of renal, as well as systemic symptoms.

In this issue of Journal of Pediatric Genetics, Bockenhauer et al. [10] report their study of 14 CLCN5 negative patients from 12 families with a phenotype resembling Dent-2 disease, for defects in OCRL1. In six of these patients, they identified three novel mutations and another three known mutations in the OCRL1 gene. None of these mutations has been described in patients with the classic Lowe syndrome. The renal phenotype of these patients was similar to that of patients harboring the CLCN5 gene mutations, except for a lower prevalence of nephrocalcinosis. 
The most interesting observation of this study is the high rate of extra-renal symptoms found in the affected children. The finding of typical symptoms of the Lowe syndrome, such as peripheral cataracts, mental impairment, growth retardation, and various degrees of elevated serum creatine kinase or lactate dehydrogenase, makes it difficult to distinguish between the two OCRL1- related syndromes. These clinical observations support previous speculations that Dent-2 disease is actually a mild variant of the Lowe syndrome [11]. It is not clear yet why some patients with OCRL1 mutations develop the complete phenotype of classic Lowe syndrome, while others develop the phenotype of Dent-2 disease [11].

The genotype/phenotype correlation in patients with OCRL1-associated diseases has not been well studied, however, there are no known mutations common to Lowe syndrome and Dent-2 disease. As shown in previous reports of OCRL1 mutations causing Dent-2 disease, two of the novel OCRL1 mutations found in this study fall into the $\mathrm{N}$-terminal half of the gene, in exons 12 and 15 and another novel mutation occurred in intron 3. All Dent-2 missense mutations fall in the phosphatidylinositol phosphate 5-phosphate domain of the Ocrl1 protein, while all of the other mutations, either nonsense or frame shift mutations, fall in the first 7 exons, and are, therefore, expected to eliminate all Ocrl1 protein function [12]. In contrast, the Lowe syndrome-associated mutations fall primarily in exons 9-22, where large functional domains map, and would be expected to reduce Ocrl1 function significantly [13].

Shrimpton et al. [12] showed that the OCRL1 mutations causing Dent-2 reside in the 5' region of the gene, and are of significantly different nature and distribution from the mutations that underlie Lowe syndrome. Analysis of a gene model revealed that splice variants in the 5' or 3' regions of OCRL1 very likely generate different isoforms of the Ocrl1 protein. They speculated that an Ocrl1-isoform initiating from the methionine in exon 8 may account for the milder phenotype in Dent-2 patients.

Interestingly, Ocrll knockout mice show no evidence of cataracts, neurological abnormalities or renal dysfunction. However, simultaneous deficiency of both the $O c r l 1$ and a highly homologous Inpp $5 b$ gene, which also encodes PIP2 5-phosphtase, results in an embryonic lethal phenotype in mice [14]. These findings suggest that Inpp5b phosphatase can compensate for the absence of the Ocrl1 enzyme in mice. Occurrence of a similar phenomenon in humans, with variable expression of a compensating enzyme among tissues and individuals, could explain the phenotypic variability in patients with OCRL1 mutations, causing either Dent-2 disease or Lowe syndrome [14].

Most recently, Bothwell et al. [15] generated mice that express human INPP5B on an Inpp5 $\mathrm{b}^{-}$and Ocrl1 ${ }^{-}$ deficient background. These mice demonstrate the common tubular abnormalities seen in Lowe syndrome and Dent-2 disease-model Ocrl1 ${ }^{-/-}$mice that express INPP5B, but not the Inpp5b protein. These mice showed reduced postnatal growth, low molecular weight proteinuria and aminoaciduria. Thus, a new animal model for Ocrll and Dent-2 disease tubulopathy was created by humanizing a modifier paralog in mice already carrying the mutant disease gene.

In conclusion, OCRL1-associate disease presents with two overlapping phenotypes: Dent- 2 and Lowe syndrome. The pathophysiology of the disease is not yet fully understood. A new mouse model may provides a tool for further investigating the mechanism by which Ocrl1 defects lead to the tubular abnormalities seen in both syndromes, and also for developing potential therapies in the future.

\section{References}

[1] Scheinman SJ. X-linked hypercalciuric nephrolithiasis: clinical syndromes and chloride channel mutations. Kidney Int 1998; 53: 3-17.

[2] Devonald MA, Karet FE. Renal epithelial traffic jams and one-way streets. J Am Soc Nephrol 2004; 15: 1370-81.

[3] Ludwig M, Doroszewicz J, Seyberth HW, Bokenkamp A, Balluch B, Nuutinen M, et al. Functional evaluation of Dent's disease-causing mutations: implications for $\mathrm{ClC}-5$ channel trafficking and internalization. Hum Genet 2005; 117: 228-37.

[4] Hoopes Jr RR, Shrimpton AE, Knohl SJ, et al. Dent disease with mutations in OCRL1. Am J Hum Genet 2005; 76: 260-67.

[5] Utsch B, Bökenkamp A, Benz MR, et al. Novel OCRL1 mutations in patients with the phenotype of Dent disease. Am J Kidney Dis 2006; 48: 942.e1-e14.

[6] Sekine T, Nozu K, Iyengar R, et al. OCRL1 mutations in patients with Dent disease phenotype in Japan. Pediatr Nephrol 2007; 22: 975-80.

[7] Tosetto E, Addis M, Caridi G, et al. Locus heterogeneity of Dent's disease: OCRL1 and TMEM27 genes in patients with no CLCN5 mutations. Pediatr Nephrol 2009; 24: 1967-73.

[8] Pendaries C, Tronchère H, Plantavid M, Payrastre B. Phosphoinositide signaling disorders in human diseases. FEBS Lett 2003; 546: 25-31.

[9] Bockenhauer D, Bokenkamp A, van't Hoff W, et al. Renal phenotype in Lowe Syndrome: a selective proximal tubular dysfunction. Clin J Am Soc Nephrol 2008; 3: 1430-36.

[10] Böckenhauer D, Bökenkamp A, Nuutinen M, et al. Novel OCRL mutations in patients with Dent-2 disease. J Pediatr Genet 2011; 15-23. 
[11] Bökenkamp A, Böckenhauer D, Cheong HI, et al. Dent-2 disease: a mild variant of Lowe syndrome. J Pediatr 2009; 155: 94-9.

[12] Shrimpton AE, Hoopes Jr RR, Knohl SJ, et al. OCRL1 mutations in Dent 2 patients suggest a mechanism for phenotypic variability. Nephron Physiol 2009; 112: 27-36.

[13] Nussbaum RL, Orrison BM, Jänne PA, Charnas L, Chinault AC. Physical mapping and genomic structure of the Lowe syndrome gene OCRL1. Hum Genet 1997; 99: 145-50.
[14] Jänne PA, Suchy SF, Bernard D, et al. Functional overlap between murine Inpp5b and Ocrl1 may explain why deficiency of the murine ortholog for OCRL1 does not cause Lowe syndrome in mice. J Clin Invest 1998; 101: 2042-53.

[15] Bothwell SP, Chan E, Bernardini IM, Kuo YM, Gahl WA, Nussbaum RL. Mouse model for lowe syndrome/dent disease 2 renal tubulopathy. J Am Soc Nephrol 2011; 22: 443-8. 